\title{
Atypical brain lateralisation in the auditory cortex and language performance in 3- to 7-year-old children with high-functioning autism spectrum disorder: a child-customised magnetoencephalography (MEG) study
}

Yuko Yoshimura ${ }^{1,2}$, Mitsuru Kikuchi ${ }^{1,2,3^{*}}$, Kiyomi Shitamichi ${ }^{2}$, Sanae Ueno ${ }^{2}$, Toshio Munesue ${ }^{1,2,3}$, Yasuki Ono ${ }^{2}$, Tsunehisa Tsubokawa ${ }^{5}$, Yasuhiro Haruta ${ }^{6}$, Manabu Oi ${ }^{1,3}$, Yo Niida $^{1}$, Gerard B Remijn ${ }^{4}$, Tsutomu Takahashi', Michio Suzuki ${ }^{7}$, Haruhiro Higashida ${ }^{1,3}$ and Yoshio Minabe $e^{1,2,3}$

\begin{abstract}
Background: Magnetoencephalography (MEG) is used to measure the auditory evoked magnetic field (AEF), which reflects language-related performance. In young children, however, the simultaneous quantification of the bilateral auditory-evoked response during binaural hearing is difficult using conventional adult-sized MEG systems. Recently, a child-customised MEG device has facilitated the acquisition of bi-hemispheric recordings, even in young children. Using the child-customised MEG device, we previously reported that language-related performance was reflected in the strength of the early component (P50m) of the auditory evoked magnetic field (AEF) in typically developing (TD) young children (2 to 5 years old) [Eur J Neurosci 2012, 35:644-650]. The aim of this study was to investigate how this neurophysiological index in each hemisphere is correlated with language performance in autism spectrum disorder (ASD) and TD children.

Methods: We used magnetoencephalography (MEG) to measure the auditory evoked magnetic field (AEF), which reflects language-related performance. We investigated the P50m that is evoked by voice stimuli (/ne/) bilaterally in 33 young children ( 3 to 7 years old) with ASD and in 30 young children who were typically developing (TD). The children were matched according to their age (in months) and gender. Most of the children with ASD were high-functioning subjects.

Results: The results showed that the children with ASD exhibited significantly less leftward lateralisation in their P50m intensity compared with the TD children. Furthermore, the results of a multiple regression analysis indicated that a shorter P50m latency in both hemispheres was specifically correlated with higher language-related performance in the TD children, whereas this latency was not correlated with non-verbal cognitive performance or chronological age. The children with ASD did not show any correlation between P50m latency and language-related performance; instead, increasing chronological age was a significant predictor of shorter P50m latency in the right hemisphere. (Continued on next page)
\end{abstract}

\footnotetext{
* Correspondence: mitsuru@zc4.so-net.ne.jp

'Research Centre for Child Mental Development, Kanazawa University,

Kanazawa, Japan

${ }^{2}$ Department of Psychiatry and Neurobiology, Graduate School of Medical

Science, Kanazawa University, Kanazawa, Japan

Full list of author information is available at the end of the article
} 
(Continued from previous page)

Conclusions: Using a child-customised MEG device, we studied the P50m component that was evoked through binaural human voice stimuli in young ASD and TD children to examine differences in auditory cortex function that are associated with language development. Our results suggest that there is atypical brain function in the auditory cortex in young children with ASD, regardless of language development.

Keywords: Autism spectrum disorder (ASD), Laterality index, Magnetoencephalography (MEG), P50m, Young children

\section{Background}

Currently, the prevalence of autism spectrum disorder (ASD) is estimated to be greater than $1 \%[1,2]$. ASD is an umbrella term for a wide variety of disorders that share common symptoms and aetiologies (i.e., autism, pervasive developmental disorder and Asperger disorder) [3]. There is a wide variety of symptoms, behaviours and types of disorders, as well as considerable individual variation. Although language impairment is not a core feature of ASD, the failure to develop sophisticated language is one of the earlier signs of this disorder [4-6].

Brain leftward lateralisation has long been one of the intriguing properties of human brain development that is associated with language acquisition [7-14]. Compared with TD children, participants with ASD have been reported to possess atypical brain lateralisation as detected by various neuroimaging methods [15-20]. From the perspective of cortical responses to speech sounds in young children or toddlers with ASD, two recent fMRI studies suggested aberrant right lateralisation by demonstrating a trend toward greater recruitment of regions of the right hemisphere during speech stimulation [18,20]. In addition, our recent study using child-sized MEG demonstrated a rightwardlateralised neurophysiological network in conscious young children (including children aged 3-4 years) with ASD while viewing a video programme with speech narration [21]. From an anatomical perspective, the rightward asymmetry of the cortical volume in the auditory association area (i.e., posterior superior temporal gyrus and planum temporale) was also shown in children with ASD [16,22]. These recent studies suggested that aberrant rightward brain function for human speech is one of the physiological hallmarks of ASD that is present at very young ages.

In studies that use magnetoencephalography (MEG), the mid-latency auditory evoked field (AEF) comprises the P50m, N100m and P200m components. The P50m $(\mathrm{P} 1 \mathrm{~m})$ is one of the mid-latency components and corresponds to the P50 (P1) in electroencephalography (EEG) studies. While the $\mathrm{P} 50(\mathrm{~m})$ has been widely used to examine sensory gating [23], the $\mathrm{P} 50(\mathrm{~m})$ is a prominent component with shorter inter-stimulus intervals (ISIs) in young children [24-27], and it can be used to provide insight into the development of auditory processing in real-world environments (i.e., auditory information at rapid rates). Although the P50m component was thought to reflect a lower level auditory processing, such as a sensory gating system [28], recent studies have demonstrated that language impairment [29] and the intervention effect [30] were also reflected in the strength of the AEFs (P50m) in young children (5 to 7 years old). In these studies, Pihko and colleagues reported that 6- to 7year-old children who have language impairments showed weaker $\mathrm{P} 50 \mathrm{~m}$ responses evoked by syllable stimuli (i.e., /da/ /ba/) compared with children who have normal language development [29]. Furthermore, they investigated the plastic changes in the AEF components in 6- to 7-year-old children after a phonological intervention programme, which comprises the following: (1) speech and articulation exercises, (2) phoneme discrimination exercises and (3) exercises that train phonological and linguistic awareness and rapid processing. Intriguingly, a significant increment in the $\mathrm{P} 50 \mathrm{~m}$ response was observed after the phonological intervention programme [30]. Furthermore, in our recent study [31], we investigated the relationships between the P50m component and several language-related subtests in the Kaufman Assessment Battery for Children (K-ABC) [32] (i.e., "number recall", "expressive vocabulary" and "riddles"). "Number recall" reflects the ability for language phonological repetition, "expressive vocabulary" reflects the expressive ability to speak the correct names of objects and illustrations and "riddles" reflects ability at language conceptual inference [32]. In our recent study, a significant correlation was found between the P50m component and performance on the "riddles", whereas no significant correlation was found with the other K-ABC language subtests. Even though the P50m component was thought to reflect a lower-level sensory processing step such as auditory input change detection for speech-like signals in adult humans [33,34], our recent study demonstrated that the $\mathrm{P} 50 \mathrm{~m}$ components were correlated with a higher-order task reflecting inferential language processing (i.e., "riddle") in preschool children [31]. These results may be explained by age-related changes in myelination, synaptic refinement and cortical fibre density [35-38], which underlie the age-related changes in amplitude and latency of $\mathrm{P} 50 \mathrm{~m}$ components. Additionally, myelination of the auditory system is followed by myelination in language-related brain areas in young children [39], which enhances a long-range brain network and 
contributes to higher-level language performance in young children. Therefore, P50m evoked by voice stimuli appeared to reflect brain maturation in association with a higher-order language development (e.g., performance of the $\mathrm{K}-\mathrm{ABC}$ subtest "riddles") in young children.

According to our previous study, the N100m component is a less detectable component that is found in MEG-equivalent current dipole (ECD) methods in 2- to 5year-old children [31]. By contrast, the P50m is a prominent component, especially during childhood [24-27], whereas $\mathrm{N} 100 \mathrm{~m}$ and $\mathrm{P} 200 \mathrm{~m}$ become more prominent after 9-10 years of age [24]. Therefore, in the present study, we focused on the P50m by using MEG ECD methods, which can yield the absolute values of the brain response in the auditory cortex.

As far as we know, no previous MEG studies have focused on this prominent component (i.e., P50m) simultaneously in both hemispheres in younger children with ASD (age 6 and under), whereas a number of auditory electrophysiological studies have primarily focused on assessing atypical auditory processing in other middletime components [e.g., N100m or mismatch field (MMF)] in older children with ASD (older than 6 years) [40-45].

A bilateral assessment of young children using simultaneous dipole source analysis revealed that AEF is severely limited for small head sizes, which has potentially restricted previous attempts at P50m studies of individuals at this age. The smaller head size of the young children certainly presents longer distances between the MEG sensors and the bilateral auditory cortices, which severely diminishes the signal-to-noise ratio because the magnetic field strength diminishes with increasing distances from the source. To overcome this problem, in the present study, we used a child-customised MEG device (PQ 1151R; Yokogawa/KIT, Kanazawa, Japan) in which the MEG sensors were positioned as close to the head as possible for optimal recording in young children, as previously reported $[7,31]$. Using this novel device, we investigated the early P50m component that was evoked through binaural human voice sounds in young ASD and TD children to examine differences in brain function in the auditory cortex of children with ASD compared to TD children. The responses that were examined are associated with language development.

To investigate childhood auditory function in realworld environments, we employed speech stimuli with a short ISI, despite the considerable refractoriness that this causes (i.e., auditory information at rapid rates), and we focussed on the values of the $\mathrm{P} 50 \mathrm{~m}$ component in the right and left hemispheres, respectively. Given that a shortened refractory period is reflected in higher P50m amplitude and shorter P50m latency, these physiological indices must represent brain maturation in developing children. We investigated how this neurophysiological index in each hemisphere is correlated with language development in young children with ASD and TD children.

\section{Methods \\ Participants}

The clinical group included 35 children with ASD (6 girls and 29 boys) at 40-93 months of age, who were recruited from Kanazawa University and prefectural hospitals in the Kanazawa or Toyama area. The ASD diagnosis was made by a psychiatrist and a clinical speech therapist. The speech therapist, who is well trained and has the Autism Diagnostic Observational Schedule research license as well as more than 5 years of experience in ASD treatment, employed the Autism Diagnostic Observational Schedule, Generic (ADOS-G) [46]. The definitive diagnosis of ASD was made by the psychiatrist, who has more than 10 years of experience in ASD, using the Diagnostic Interview for Social and Communication Disorders (DISCO) [47] and the DSM-IV criteria (the American Psychiatric Association, 1994) at the time of the MEG and the Kaufman Assessment Battery for Children (K-ABC) data acquisition. All the children with ASD included in this study satisfied the diagnosis of childhood autism $(n=25)$, atypical autism $(n=4)$ or Asperger's syndrome $(n=6)$ using the DISCO. Children who were below the ADOS cutoff levels were included in the present study if they met the criteria for ASD using both the DSM-IV criteria and DISCO (i.e., 6 children). A total of 35 TD children ( 6 girls and 29 boys) ranging from 37 to 85 months old, with no reported behavioural or language problems, were used as controls. The TD children were matched to the ASD subjects according to age in months. All the TD subjects were native Japanese children with no previous or existing developmental, learning or behavioural problems according to information obtained from a questionnaire completed by their parents. All the participants had normal hearing abilities according to available medical records. Left- or right-hand dominance was determined based on their preference when handling objects, and the following results were obtained: TD children (right $=35$, left $=0$, both $=0$ ) and children with ASD (right $=27$, left $=2$, both $=6$ ). There were no significant differences in head size between the two groups.

All of the children participated separately in cognitive tasks and MEG measurements over a 2-day period. On the first day, the participants performed cognitive tests and were introduced to the environment for the MEG measurement. The actual MEG measurements were performed on the second day. The parents agreed to the participation of their child in the study with full knowledge of the experimental nature of the research. Written informed consent was obtained prior to participation in the study. The Ethics Committee of Kanazawa University 
Hospital approved the methods and procedures, all of which were performed in accordance with the Declaration of Helsinki.

\section{Cognitive and language performance measurements}

The children were subjected to the Japanese adaptation of the Kaufman Assessment Battery for Children (K-ABC), which is typically used to assess the cognitive skills of children aged 30-155 months. To confirm the standardised score of the mental processing and achievement scales in children, subtests that were complementary to the age (in months) of the children were used in this battery. Because we have demonstrated the significant correlation between the early component of the AEF (i.e., the P50m intensity) and the performance of a language-related task (i.e., a subtest of $\mathrm{K}-\mathrm{ABC}$ 'riddles') in our previous study [31], we employed this subtest 'riddles' in this study. In the riddle task, children were required to answer the examiner's question, such as "Which fruit has a rounded shape with a depression at the top where the stem is attached? The colour of the skin can be either red, green, yellow, or a combination of these colours". In this case, the answer is "an apple". The riddle task consists of 32 questions, which are presented in ascending order of difficulty. The linguistic level is defined by the child's degree of achievement. The K-ABC 'riddles' subtest reflects conceptual language inference abilities [32], and our recent study demonstrated that 'riddles' was the weakest subtest for the children with ASD compared with the TD children [48]. There were no significant differences in the $\mathrm{K}-\mathrm{ABC}$ mental processing scale/achievement scale between groups (Table 1).

\begin{tabular}{|c|c|c|c|c|}
\hline & TD & ASD & $t$ & $p$ \\
\hline Number of subjects & 33 & 30 & & \\
\hline Gender (male/female) & $27 / 6$ & $25 / 5$ & & \\
\hline Chronological age (months) & $67.4(10.7)$ & $66.9(12.0)$ & 0.15 & n.s. \\
\hline Head size $(\mathrm{cm})$ & $51.5(1.5)$ & $51.0(1.8)$ & 1.23 & n.s. \\
\hline Handedness (right) & 33 & 24 & & \\
\hline \multicolumn{5}{|l|}{$K-A B C$} \\
\hline Mental Processing Scale & $97.4(12.8)$ & $94.3(18.9)$ & 0.75 & n.s. \\
\hline Achievement Scale & $96.2(12.2)$ & $95.3(21.8)$ & 0.21 & n.s \\
\hline \multicolumn{5}{|l|}{ ADOS } \\
\hline Communication & - & $3.9(2.1)$ & & \\
\hline Reciprocal Social Interaction & - & $7.2(2.3)$ & & \\
\hline
\end{tabular}

K-ABC, Kaufman Assessment Battery for Children. ADOS, Autism Diagnostic Observation Schedule.

The values are the mean (standard deviation) for chronological age, head size, scales on the K-ABC and scores of ADOS.

n.s., no significance.

\section{Magnetoencephalography recordings}

The conditions used were similar to those detailed in our previous study [31]. The MEG data were recorded using a 151-channel SQUID (Superconducting Quantum Interference Device) whole-head coaxial gradiometer MEG system for children (PQ 1151R; Yokogawa/KIT, Kanazawa, Japan; Figure 1) in a magnetically shielded room (Daido Steel, Nagoya, Japan), which was installed at the MEG Centre of Yokogawa Electric Corp. (Kanazawa, Japan). The custom child-sized MEG system facilitates the measurement of brain responses in young children, which would be difficult to obtain using conventional adult-sized MEG systems. The child MEG system ensures that sensors are easily and effectively positioned within range of the brain and that head movement is constrained [49]. We determined the position of the head within the helmet by measuring the magnetic fields after passing currents through coils attached at three locations on the head surface, which were used as fiduciary points with respect to the landmarks (bilateral mastoid processes and nasion). Although we could not determine whether the individual head shape would influence the accuracy of the dipole estimation, for calculating ECD without magnetic resonance imaging anatomical data, a spherical model of the volume conductor was fitted to the head of each subject and was confirmed to be located in the centre of the MEG helmet through measurements at three locations on the head surface, as described in our previous study [31]. An examiner remained in the room to encourage the children and to prevent movement throughout the analysis. Stimuli were presented while the children lay in the supine position on the bed and viewed silent video programmes projected onto a screen.

\section{Auditory evoked field stimuli and procedures}

MEG recordings were obtained from all participants during auditory syllable sound stimulation that comprised the Japanese syllable /ne/ [31]. We employed this syllable because /ne/ is one of the Japanese final sentence particles that carry prosodic information [50,51]. The syllable /ne/ is often used in Japanese mother-child conversations and expresses the speaker's request for acknowledgement or empathy from the listener [52,53]. In the present study, we used typical oddball sequences consisting of standard stimuli at a rate of $83 \%$ (456 times) and deviant stimuli at a rate of $17 \%$ (90 times). In the standard stimulus, /ne/ was pronounced with a steady pitch contour, whereas in the deviant condition, /ne/ was presented with a falling pitch. Eventually, we adopted only standard stimuli for subsequent ECD estimations because a sufficient number of periods to calculate ECD remained after the rejection of artefacts in all the children. A female native Japanese speaker produced the /ne/ sounds, which were presented using a condenser microphone (NT1-A; 

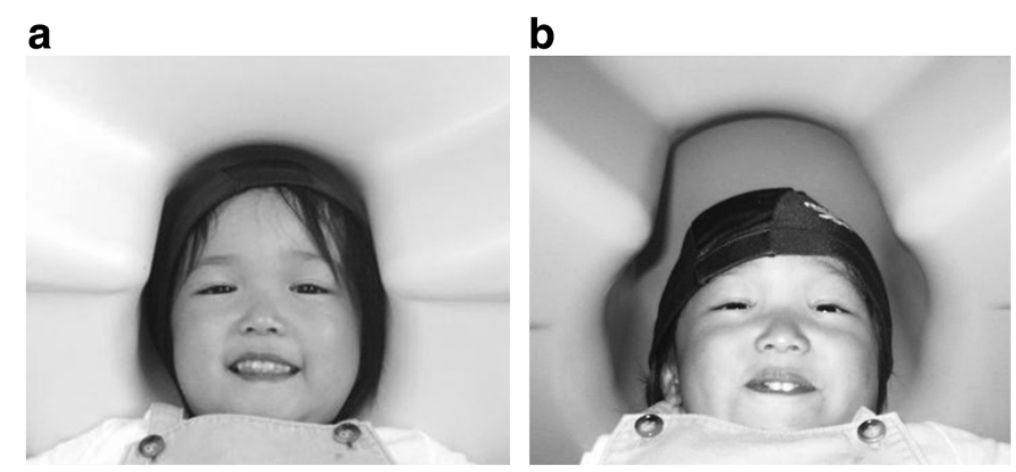

Figure 1 Comparison between child-sized MEG and adult-sized MEG for head size of young children. (a) A customised, child-sized MEG. (b) A conventional, adult-sized MEG.

Rode, Silverwater, NSW, Australia) on a personal computer. As shown in Figure 2, the duration of the stimulus was $342 \mathrm{~ms}$, and the duration of the consonant /n/ was 65 ms. In this study, the beginning of the vowel sound /e/ was defined as the onset time. The ISI was $818 \mathrm{~ms}$. Both stimuli had a level of approximately $65 \mathrm{~dB}$ (A-weighted) against a background noise of $43 \mathrm{~dB}$, which was measured with an integrating sound level meter (LY20; Yokogawa, Tokyo, Japan). The stimulus was presented to participants binaurally through a hole in the MEG chamber using speakers (HK195 Speakers; Harman Kardon, Stamford,

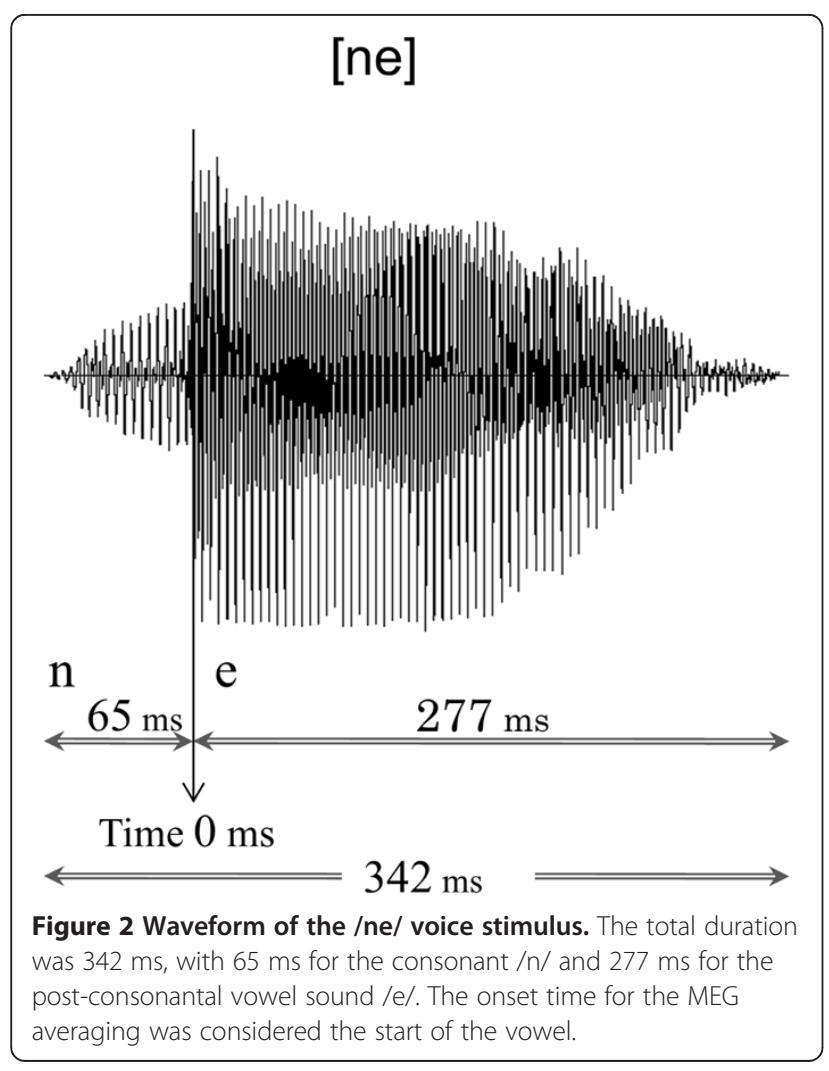

CT) that were placed outside of the shielded room. The duration of the recording was $12 \mathrm{~min}$.

\section{AEF acquisition and analysis}

The participant's head was placed in a whole-head Dewar that contained 151 concentrically arranged magnetic sensors. The MEG data were acquired with a sampling rate of $1,000 \mathrm{~Hz}$ and were filtered using a 200-Hz low-pass filter. The time series obtained started from the onset of the syllable stimulus at $-150 \mathrm{~ms}$ and continued to $1,000 \mathrm{~ms}$, and subsequent segments (at least 300 for standard stimuli) were averaged for each of the sensors after baseline correction (-50 to $0 \mathrm{~ms}$ ) (Figure 3a, b). The segments that were contaminated with artefacts (eye-blink and eye and body movements, typically more than $\pm 4 \mathrm{pT}$ ) were excluded from the analysis. A single ECD model was used to estimate the current sources in the activated cerebral cortex using more than 30 sensors for each hemisphere (left and right) [54]. To estimate the localisation of the current sources, MegLaboratory 160 (Yokogawa/KIT, Kanazawa, Japan) was used.

To identify the P50m component, we accepted estimated ECDs when (1) the goodness of fit (GOF) exceeded $80 \%$; (2) the location of the estimated dipoles using a single ECD model was stabilised within $\pm 5 \mathrm{~mm}$ of each coordinate for at least $6 \mathrm{~ms}$ during the target response period; (3) the dipole intensities were $\leq 80 \mathrm{nAm}$ and (4) the direction of the estimated ECD was in an anterosuperior orientation. The latency time point was defined as the maximum estimated dipole intensity value obtained in accordance with the above criteria within a time window of 40 to $150 \mathrm{~ms}$. Regarding the coordinate axes of the P50m component, the centre of a sphere in a spherical model of the volume conductor for the ECD estimation was defined as the origin, and the $x-, y-$ and $\mathrm{z}$-axes represent the leftward direction, the occipital direction and the vertex, respectively. 


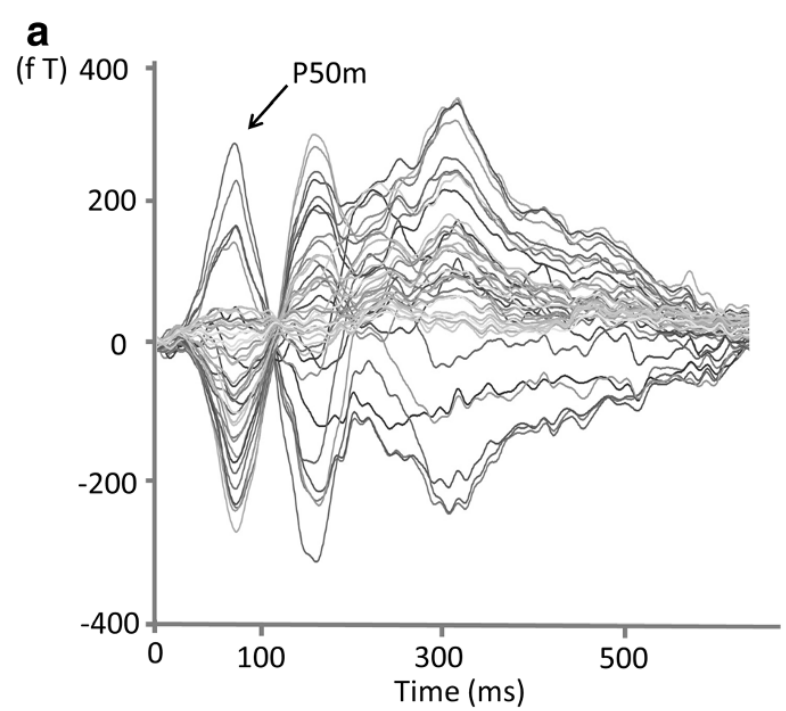

\section{b}
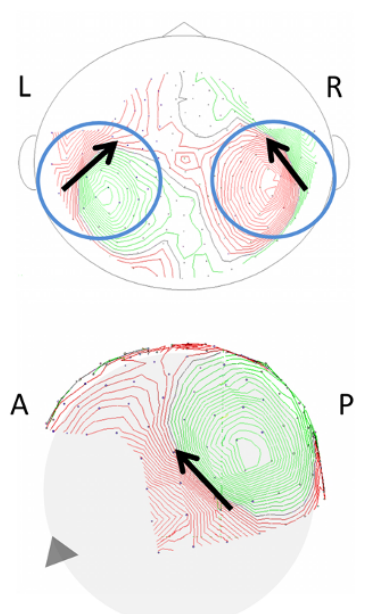

Figure 3 Source modelling of neuromagnetic responses evoked through auditory stimuli. (a) The response showed an activity peak at 45-150 ms. (b) Typical magnetic field patterns of the peak in the auditory cortex. The magnetic field strength is indicated by a coloured line, which varies from green (flux-in) to red (flux-out). Sensor locations are indicated by small dots. Black arrows indicate the directions of equivalent current dipole orientation. Blue open circles indicate the examples of the 30 sensors for each hemisphere (left and right) to estimate the current sources. $A$, anterior; $P$, posterior; $L$, left; $R$, right.

\section{Data analysis}

Using the Kolmogorov-Smirnov test, it was confirmed that each physiological variable (i.e., raw value of latency and log-transformed intensity) and the scores on the language subtests were normally distributed $(p>0.05)$. For each physiological variable (the log-transformed intensity and latency), a two-way ANOVA was performed (subject group $\times$ hemisphere). The between-subject variable was the group (TD vs. ASD), and the within-subject variable was the hemisphere (left and right). Statistical significance was defined as $p<0.05$. The estimated dipole position of the P50m was also compared for each axis (i.e., the $\mathrm{x}, \mathrm{y}$ and $\mathrm{z}$ directions) between the two groups using a two-way ANOVA (subject group $\times$ hemisphere). The between-subject variable was the group (TD vs. ASD), and the within-subject variable was the hemisphere (left and right). For the $\mathrm{X}$ direction (the positive and negative values in the row data corresponding to the left and right directions, respectively), the absolute value was used for this comparison. The alpha level was adjusted to $0.05 / 3=0.017$ for the statistical analysis because of the multiple comparisons in three axes (i.e., $\mathrm{x}, \mathrm{y}$ and $\mathrm{z}$ ).

A hierarchical regression analysis was used to investigate the association between the $\mathrm{P} 50 \mathrm{~m}$ component (i.e., the log-transformed intensity and latency) and the languagerelated subscore (i.e., the performance of 'riddles'). Analyses were performed separately for the right and left hemispheres. Between-person covariates were included in the hierarchical regression analysis as independent factors that were of theoretical importance in a study of brain development and cognitive function. These variables were age (in months) and non-verbal performance (i.e., a combined score of the $\mathrm{K}-\mathrm{ABC}$ subtests in 'face recognition' and 'hand movement').

To investigate whether there is a significant change in the explained variance of the model that includes the final independent factor (i.e., the performance of 'riddles') compared to the model that includes only the confounding variables (age in months and non-verbal performance), we employed a hierarchical regression model in which the language task performance was included in the final step. The demographic variable (age in months) was entered at the first step in this model. The non-verbal performance was entered at the second step, and the language-related subscore was entered at the final step. The alpha level was adjusted to $0.05 / 2=$ 0.025 for the statistical analysis of the P50m component because of the multiple comparisons in the two hemispheres (i.e., left and right).

\section{Results}

The profile of the K-ABC subscores in children with ASD and TD children.

When we compared the performances on the K-ABC subtests that were common to all ages between the TD children $(n=35)$ and the children with ASD $(n=35)$ (Additional file 1: Figure S1), an unpaired t-test revealed that "riddles" (the verbal reasoning task) was the weakest subtest for the children with ASD $(t=2.285 ; p=0.025)$. 
In terms of the scores of the "riddles" (the verbal reasoning task), there were no significant differences between children who achieved a full ADOS score $(n=29)$ and children who did not achieve a full ADOS score $(n=6)$ $(t=-0.800 p=0.429)$.

\section{Detection of the P50m component}

One of the 35 children with ASD did not complete the experiment because of boredom and unwillingness to undergo the measurements. Therefore, the MEG data were acquired from 35 TD children and 34 children with ASD. The P50m component could not be detected in one or both hemispheres in two TD children and four children with ASD (i.e., did not meet the criteria for ECD). In total, the bilateral ECD sources were reliably estimated from 33 TD children and 30 children with ASD. Demographic characteristics are shown in Table 1 for the participants whose P50m dipole sources were reliably estimated in both hemispheres.

\section{P50m intensity}

For the TD children, the dipole intensity was $21.6 \pm 8.8$ and $13.7 \pm 6.9 \mathrm{nAm}$ (mean $\pm \mathrm{SD}$ ) in the left and right hemispheres, respectively. For the children with ASD, the dipole intensity was $17.2 \pm 7.8$ and $14.4 \pm 5.6$ (mean $\pm \mathrm{SD}$ ) in the left and right hemispheres, respectively. A comparison of the log-transformed intensity using two-way ANOVA (subject group $\times$ hemisphere) revealed a significant main effect of the hemisphere ( $\mathrm{F}=22.36 ; p<0.0001$ ); however, there were no significant effects of the group. Significant interactions between the group and hemisphere were observed $(\mathrm{F}=7.54 ; p=0.007$, Figure $4 \mathrm{a})$. This significant interaction indicates that the children with ASD exhibited significantly less leftward lateralisation compared with the TD children.

As a complementary analysis, we re-calculated the two-way ANOVA (subject group $\times$ hemisphere) in only the right-handed participants $(\mathrm{TD}=33, \mathrm{ASD}=24)$ to exclude the confounding effect of handedness. Almost the same results were obtained: there was a significant main effect of the hemisphere ( $\mathrm{F}=17.52 ; p=0.0001)$, there were no significant effects of the group, and significant interactions between the group and the hemisphere were observed ( $\mathrm{F}=7.54 ; p=0.0008)$.

In addition, as a complementary analysis, we re-calculated the two-way ANOVA (subject group $\times$ hemisphere) without the six children with ASD who did not meet the criteria of ASD by ADOS (TD = 33, ASD = 25). Almost the same results were obtained: a significant main effect of the hemisphere ( $\mathrm{F}=19.67 ; p<0.0001)$ was observed. There were no significant effects of the group $(\mathrm{F}=0.56 ; p=0.456)$. Significant interactions between the group and hemisphere were observed ( $\mathrm{F}=5.88 ; p=0.018)$.

\section{P50m Latency}

For the TD children, the mean dipole latency was $88 \mathrm{~ms}$ (1 SD = 13) and $81 \mathrm{~ms}(1 \mathrm{SD}=13)$ in the left and right hemispheres, respectively. For the children with ASD, the mean dipole latency was $87 \mathrm{~ms}(1 \mathrm{SD}=19)$ and 82 $\mathrm{ms}(1 \mathrm{SD}=18)$ in the left and right hemispheres, respectively. A comparison of the latency using two-way ANOVA (subject group $\times$ hemisphere) revealed a significant main effect of the hemisphere $(\mathrm{F}=9.702 ; p=$ 0.002); however, no significant main effects of the group were observed. Moreover, no significant interactions between the group and the hemisphere were observed ( $\mathrm{F}=0.434 ; p=0.512$; Figure $4 \mathrm{~b})$.

As a complementary analysis, we re-calculated the two-way ANOVA (subject group $\times$ hemisphere) in only the right-handed participants (TD $=33, \mathrm{ASD}=24)$ to exclude the confounding effect of the handedness. Almost the same results were obtained: there was a significant main effect of the hemisphere $(\mathrm{F}=7.835 ; p=0.008)$, no significant main effect of the group was observed, and
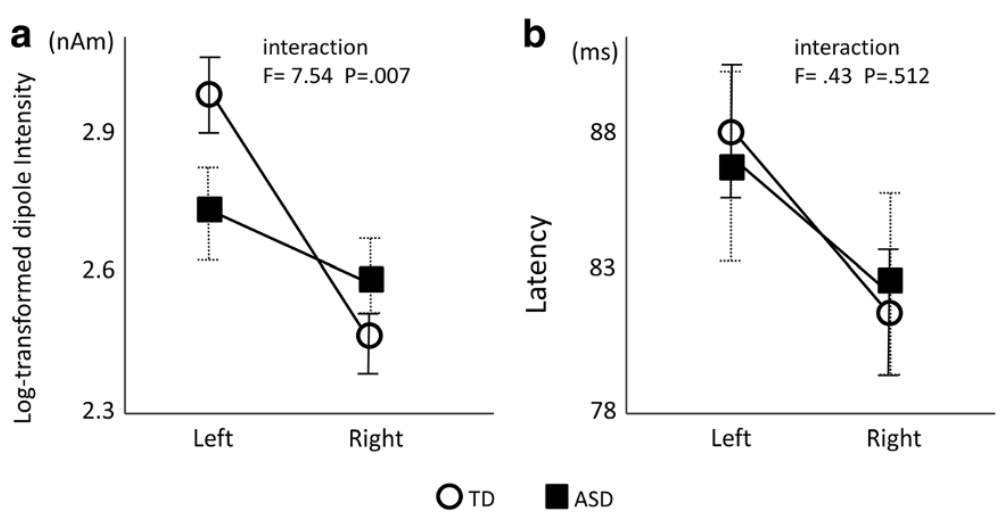

Figure 4 Intensity (a) and latency (b) of the P50m component. The open circles indicate typically developing children, and the closed squares indicate children with ASD. Note the significant interaction between the group and hemisphere ( $F=7.54 ; p=0.007)$ for the intensity values (a) but not for the latency values (b). The values indicate the mean $\pm \mathrm{SE}$. 
no significant interactions between the group and hemisphere were observed ( $\mathrm{F}=0.722 ; p=0.399)$.

In addition, as a complementary analysis, we recalculated the two-way ANOVA (subject group $\times$ hemisphere) without the six children with ASD who did not meet the criteria of ASD by ADOS (TD = 33, ASD = 25). Almost the same results were obtained: there was a significant main effect of the hemisphere ( $\mathrm{F}=10.47 ; p=$ 0.002); however, no significant main effects of the group were observed. Moreover, no significant interactions between the group and the hemisphere were observed $(\mathrm{F}=0.09 ; p=0.764)$.

\section{Source localisation of the P50m}

The results of the two-way ANOVA (subject group $x$ hemisphere) showed a significant main effect of the hemisphere in the $\mathrm{x}-(\mathrm{F}=10.626 ; p=0.0018), \mathrm{y}-(\mathrm{F}=$ 63.447; $p<0.0001)$ and $\mathrm{z}$-axes $(\mathrm{F}=16.539 ; p=0.001)$ (Table 2). However, there was no significant main effect of the group in the $\mathrm{x}-, \mathrm{y}$ - and $\mathrm{z}$-axes, and no significant interaction was observed between the group and the hemisphere in the $\mathrm{x}-(\mathrm{F}=0.064 ; p=0.801), \mathrm{y}-(\mathrm{F}=$ $2.653 ; p=0.987)$ and $z$-axes $(\mathrm{F}=3.159 ; p=0.080)$.

\section{Correlation between P50m intensity and language-related performance}

Hierarchical regression analyses examining the relationship between the $\mathrm{P} 50 \mathrm{~m}$ log-transformed intensity (in the right and left hemispheres) and the performance of the K-ABC language subtest 'riddles' were conducted separately for the TD children and ASD children. As shown in Table 3 , in both the TD children and ASD children, the hierarchical multiple regression model revealed no significant relationships between the $\mathrm{K}-\mathrm{ABC}$ 'riddle' subtest performance and the P50m dipole intensity at any step. However, in the TD children, the 'nonverbal performance' reached statistical significance at steps 2 and 3 (final step $3, \beta=0.751, p=0.008$ ) in the right hemisphere (Table 3) (an increase in the P50m intensity was correlated with an increased nonverbal performance in the final step). Age was not a statistically significant factor at any step (Table 3 ) in either the TD children or the ASD children.

As a complementary analysis, we re-calculated the hierarchical regression analysis except six children with ASD who did not meet the criteria of ASD by ADOS
$($ ASD $=25)$. Almost the same results were obtained: no independent factors reached significance at any step (Additional file 1: Table S1) in children with ASD.

\section{Correlation between P50m latency and language-related performance}

A hierarchical regression analysis examining the relationship between the P50m latency (in the right and left hemispheres) and the performance on the K-ABC language subtest 'riddles' was conducted separately for the TD children and ASD children. As shown in Table 4, in the TD children, the hierarchical multiple regression model revealed that the $\mathrm{K}-\mathrm{ABC}$ 'riddle' language subtest performance was significantly and independently associated with the P50m latency in both the left (final step 3, $\left.\Delta \mathrm{R}^{2}=0.326, \beta=-0.521, p=0.012\right)$ and right hemispheres (final step $3, \Delta \mathrm{R}^{2}=0.255, \beta=-0.577, \mathrm{p}=0.009$ ) (a decrease in the $\mathrm{P} 50 \mathrm{~m}$ latency was correlated with higher verbal performance in the final step), whereas in the children with ASD, no significant relationships between the K-ABC 'riddle' subtest performance and the P50m latency were demonstrated at any step.

In addition, for the TD children, there was a significant relationship between the P50m latency in the left hemisphere and the age in months during the first two steps; however, this significance diminished at step 3 (Table 4). In the right hemispheres of the children with ASD, a relationship between $\mathrm{P} 50 \mathrm{~m}$ latency and age maintained statistical significance until the final step (step $3, \beta=-0.583, p=0.005$ ) (i.e., a decrease in the P50m latency was correlated with a higher age in months in the final step). The relationship between the P50m latency and the 'non-verbal performance' did not reach statistical significance at any step (Table 4) in both the TD children and ASD children.

As a complementary analysis, we re-calculated the hierarchical regression analysis without the six children with ASD who did not meet the criteria of ASD by ADOS (ASD = 25). Although the same tendency was observed, the relationship between P50m latency and age was diminished at all steps (Additional file 1: Table S2), and no independent factor reached significance at any step (Additional file 1: Table S2) in children with ASD.

In addition, as a complementary analysis for the relationships in which the significance was observed at the final step in the first analysis (TD $=33$, ASD $=25$ ),

Table 2 Position of the P50m source

\begin{tabular}{ccccccc}
\hline & \multicolumn{3}{c}{ Left } & & \multicolumn{2}{c}{ Right } \\
\hline \multirow{2}{*}{ TD } & $x$ & $y$ & $z$ & $x$ & $y$ & $z$ \\
ASD & $51.61(7.6)$ & $3.77(6.0)$ & $-7.85(8.6)$ & $-56.80(8.3)$ & $-7.66(8.8)$ & $-16.35(16.3)$ \\
\hline
\end{tabular}

The centre of the hypothetical spherical head model is the origin. The values are the mean (standard deviation).

The positive values on the $x^{-}, y$ - and $z$-axes indicate the leftward direction, the occipital direction and vertex, respectively. 
Table 3 Summary of the hierarchical regression analysis for P50m log-transformed intensity

\begin{tabular}{|c|c|c|c|c|c|c|c|c|c|c|}
\hline & \multirow{2}{*}{\multicolumn{4}{|c|}{$\begin{array}{c}\text { Left } \\
\beta\end{array}$}} & \multirow{2}{*}{\multicolumn{4}{|c|}{$\begin{array}{c}\text { Right } \\
\beta\end{array}$}} & \multirow[t]{3}{*}{$\mathrm{N}$} \\
\hline & & & & & & & & & & \\
\hline & & Step 1 & Step 2 & Step 3 & $t$ in step 3 & Step 1 & Step 2 & Step 3 & $t$ in step 3 & \\
\hline \multirow[t]{6}{*}{ TD } & Age (months) & 0.172 & 0.097 & 0.108 & 0.331 & 0.067 & -0.532 & -0.592 & -2.036 & 33 \\
\hline & Non-verbal performance & & 0.095 & 0.097 & 0.327 & & $0.763^{*}$ & $0.751^{*}$ & 2.846 & \\
\hline & Riddles & & & -0.018 & -0.075 & & & 0.104 & 0.479 & \\
\hline & $\mathrm{R}$ & 0.172 & 0.182 & 0.182 & & 0.067 & 0.477 & 0.483 & & \\
\hline & Adjusted $R^{2}$ & 0.030 & 0.033 & 0.033 & & 0.004 & 0.227 & 0.233 & & \\
\hline & $\Delta R^{2}$ & 0.000 & 0.000 & 0.000 & & 0.000 & 0.176 & 0.154 & & \\
\hline \multirow[t]{6}{*}{ ASD } & Age (months) & -0.051 & 0.013 & -0.061 & -0.284 & -0.288 & -0.246 & -0.294 & -1.390 & 30 \\
\hline & Non-verbal performance & & -0.292 & -0.392 & -1.692 & & -0.187 & -0.251 & -1.096 & \\
\hline & Riddles & & & 0.197 & 0.761 & & & 0.126 & 0.498 & \\
\hline & $\mathrm{R}$ & 0.051 & 0.289 & 0.322 & & 0.288 & 0.340 & 0.352 & & \\
\hline & Adjusted $R^{2}$ & 0.003 & 0.084 & 0.104 & & 0.083 & 0.116 & 0.124 & & \\
\hline & $\Delta R^{2}$ & 0.000 & 0.016 & 0.000 & & 0.050 & 0.050 & 0.023 & & \\
\hline
\end{tabular}

${ }^{*} p<0.025$

Pearson's correlation coefficients (i.e., simple linear regression) were calculated separately between the $\mathrm{P} 50 \mathrm{~m}$ latency and the verbal performance (Figure 5) or the age in months (Figure 6) in the left and right hemispheres in the TD children and ASD children. As shown in Figure 5a and b, a significant negative correlation between the P50m dipole latency and the verbal performance was observed in the left $(\mathrm{r}=-0.586, p<0.001)$ and the right $(r=-0.479$, $p=0.005$ ) hemispheres in the TD children, whereas no significant correlation was observed in the children with ASD in either hemisphere. As shown in Figure $6 a$ and b, a significant negative correlation between P50m dipole latency and age in months was observed in the left hemisphere of the TD children $(r=-0.463, p=0.007)$, whereas this negative correlation was observed in the right hemisphere of the children with ASD $(r=-0.435, p=0.016)$.

\section{Discussion}

This study is the first report to describe the features of bilateral auditory evoked field (AEF) dipole source features in young children (3-7 years old) with ASD. Using MEG devices customised for children, we replicated our previous study for 2 - to 5 -year-old TD children $(n=59$; five participants were the same as in the present study [31]), which demonstrated leftward lateralisation in the P50m dipole intensity during binaural human voice stimulation in TD children. In addition, our present result revealed that the children with ASD showed less

Table 4 Summary of the hierarchical regression analysis for P50m latency

\begin{tabular}{|c|c|c|c|c|c|c|c|c|c|c|}
\hline & & \multicolumn{4}{|c|}{$\begin{array}{c}\text { Left } \\
\beta\end{array}$} & \multicolumn{4}{|c|}{$\begin{array}{c}\text { Right } \\
\beta\end{array}$} & \multirow[t]{2}{*}{$\mathbf{N}$} \\
\hline & & Step 1 & Step 2 & Step 3 & $t$ in step 3 & Step 1 & Step 2 & Step 3 & $t$ in step3 & \\
\hline \multirow[t]{6}{*}{ TD } & Age (months) & $-0.463^{*}$ & $-0.657^{*}$ & -0.358 & -1.380 & -0.267 & -0.599 & -0.268 & -0.982 & 33 \\
\hline & Non-verbal performance & & 0.248 & 0.307 & 1.302 & & 0.423 & 0.488 & 1.972 & \\
\hline & Riddles & & & $-0.521^{*}$ & -2.681 & & & $-0.577^{*}$ & -2.823 & \\
\hline & $\mathrm{R}$ & 0.463 & 0.487 & 0.624 & & 0.267 & 0.374 & 0.570 & & \\
\hline & Adjusted $\mathrm{R}^{2}$ & 0.214 & 0.237 & 0.389 & & 0.071 & 0.140 & 0.325 & & \\
\hline & $\Delta R^{2}$ & 0.189 & 0.187 & 0.326 & & 0.041 & 0.082 & 0.255 & & \\
\hline \multirow[t]{6}{*}{ ASD } & Age (months) & -0.058 & -0.100 & -0.122 & -0.553 & $-0.435^{*}$ & $-0.503^{*}$ & $-0.583^{*}$ & -3.092 & 30 \\
\hline & Non-verbal performance & & 0.192 & 0.162 & 0.677 & & 0.308 & 0.199 & 0.974 & \\
\hline & Riddles & & & 0.058 & 0.218 & & & 0.214 & 0.935 & \\
\hline & $\mathrm{R}$ & 0.058 & 0.196 & 0.201 & & 0.435 & 0.528 & 0.550 & & \\
\hline & Adjusted $\mathrm{R}^{2}$ & 0.003 & 0.038 & 0.040 & & 0.189 & 0.279 & 0.302 & & \\
\hline & $\Delta R^{2}$ & 0.000 & 0.000 & 0.000 & & 0.160 & 0.226 & 0.222 & & \\
\hline
\end{tabular}




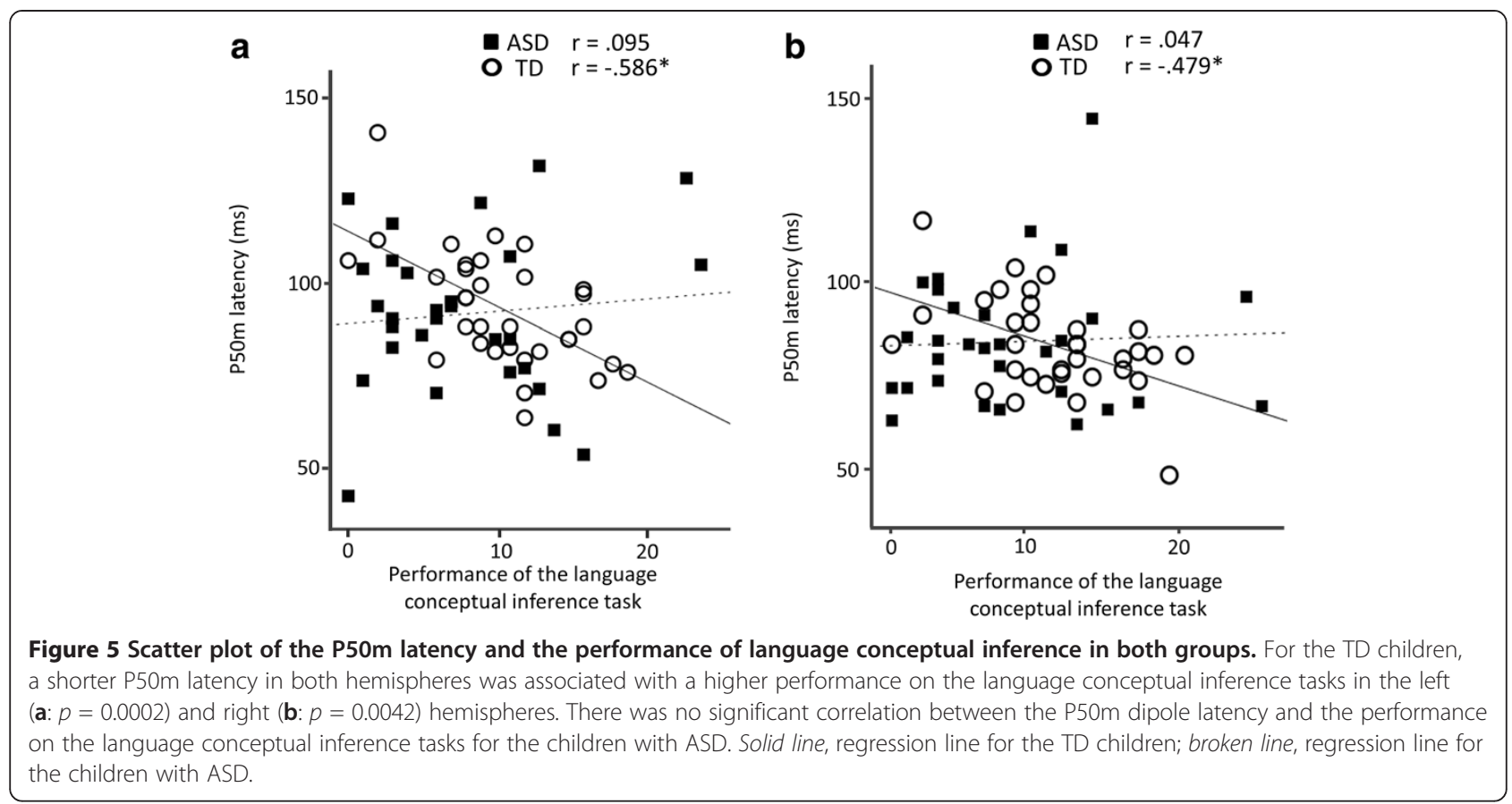

leftward lateralisation in the intensity of the P50m during short ISI human voice stimuli compared with TD children. Interestingly, as a result of the multiple regression analysis to investigate the relationship between the P50m component and language performance, a shorter P50m latency in both hemispheres was specifically correlated with higher language-related performance in the TD children. This result suggested that the human voice evoked the P50m component in both hemispheres, as reflected by the brain maturation in structures related to language acquisition. Given that myelination considerably enhances nerve conduction velocity and leads to a shorter latency of the brain response [55-58], a shorter P50m latency to human voice stimuli might reflect the progression of increasing myelination in the brain areas related to human voice processing. In contrast, the P50m latency in children with ASD did not show any correlation with language-related performance.

Functional brain organisation is thought to be shaped in an activity-dependent fashion by a complex interaction between multiple genes and epigenetic factors [59]. The language processing area is one of the most interesting
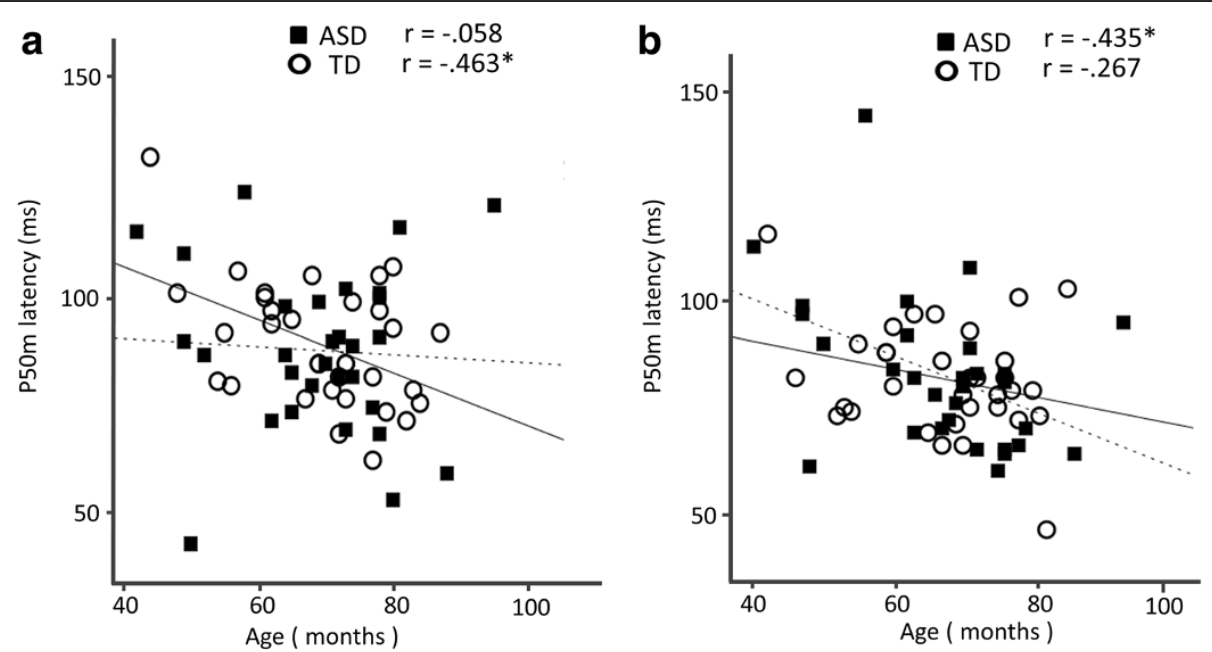

Figure 6 Scatter plot of the P50m dipole latency and age, in both groups. For the TD children, a shorter P50m latency in the left hemisphere was associated with a higher age (a: $p=0.0061)$. By contrast, for the children with ASD, a shorter P50m latency in the right hemisphere was associated with a higher age $(\mathbf{b}: p=0.0156)$. Solid line, regression line for the TD children; broken line, regression line for children with ASD. 
regions in which a correlation between myelination and language ability has been reported in young children [39]. Our results suggest that auditory-related brain organisation in TD children is shaped in a language-abilitydependent fashion that is also affected by chronological age. In children with ASD, however, we failed to demonstrate an ability dependence of the $\mathrm{P} 50 \mathrm{~m}$ component. This result suggests that, in the right hemisphere, auditoryrelated brain organisation in ASD is shaped in a languageability-independent fashion while being affected by chronological age. Although further study is necessary to demonstrate what type of ability is associated with the P50m component in children with ASD, this languagerelated functional discrepancy may shed light on the neural basis of ASD.

In the present study, increasing chronological age was associated with shorter P50m latency in the right hemisphere in ASD but not in TD children, from a hierarchical regression analysis. As reported in the previous EEG study, with 118 normal subjects, the P1 (i.e., P50m in the MEG study) latency decreases with age between 5 and 20 years of age [24], and it therefore appears to be natural for both TD and ASD children that increasing chronological age is associated with shorter P50m latency. In the present study, no statistical significance of the regression analysis in TD children could be attributed to the narrower range in the age (i.e., 3 to 7 years old), whereas significant results observed in children with ASD may be due to the accelerated maturation of white matter in young children with ASD within such a narrow age range [60].

In contrast to the TD children, the present results revealed that children with ASD showed a less leftlateralised P50m during human voice stimuli (Figure 4a). This atypical brain lateralisation during human voice stimuli is consistent with recent MEG studies in older children with ASD (8-17 years old) [61]. These authors demonstrated rightward lateralisation in the AEF late component during vowel stimuli in relatively small samples $(n=6)$, which suggests that children with ASD and TD children follow opposite maturational trajectories in language-related brain lateralisation. Our results provide further evidence in younger children with ASD during conscious conditions. In addition, our results (less leftward lateralisation in children with ASD) were supported by those obtained from recent brain volumetric studies using MRI, which revealed an aberrant rightward lateralisation $[15,16,22,62]$. Intriguingly, the rightward asymmetry of the cortical volume in the auditory association area (i.e., posterior superior temporal gyrus and planum temporale) was shown in children with ASD $[16,22]$. Thus, these results are consistent with our results and showed less leftward lateralisation in the P50m intensity, which is generated in these areas [63].
In the correlation analysis for P50m intensity and language performance, our current study failed to demonstrate a significant correlation, which we had previously found in 2- to 5-year-old children [31]. The discrepancy between these studies might reflect the older and wider age range of the subjects (3 to 7 years old) in the present study. Previous EEG studies demonstrated that the growth curve of the P50 amplitude (but not the latency) in young children followed an inverted U-shaped curve. These authors reported that the amplitude of the P50 was the largest in toddlers ( 1 to 2 years old) compared with newborns ( $<7$ days) and young children ( 4 to 6 years old) [27] and that it decreased with age from childhood to adulthood $[24,64]$. Judging from these results, linear regression models are not always suitable for comparing the P50m intensity and age in young children. Incidentally, in the present study, an unexpected association between the P50m dipole intensity in the right hemisphere and non-verbal performance was also demonstrated in TD children. However, caution must be exercised in drawing any definitive conclusions from this multiple regression model using the P50m intensity and the age in months because of the same reasons mentioned above (i.e., linear regression models might not be suitable).

Although no previous MEG study has focused on the P50m in preschool ( $<7$ years old) children with ASD, there are a few P50m studies in school-age children and adolescents with ASD $[25,42,65]$. One previous study investigated the relationship between clinical language impairment and the latency of auditory $\mathrm{P} 50 \mathrm{~m}$ and $\mathrm{N} 100 \mathrm{~m}$ in 7- to 18-year-old subjects with various clinical conditions (i.e., typical development, ASD or specific language impairment) and demonstrated that longer P50m latency in the right hemisphere was the best predictor of clinical language impairment [65]. However, we observed similar relationships (i.e., longer $\mathrm{P} 50 \mathrm{~m}$ latency correlated with lower language performance) in both hemispheres in the TD children but not in the children with ASD. The other study investigated the $\mathrm{P} 50 \mathrm{~m}$ and $\mathrm{N} 100 \mathrm{~m}$ only in the left hemisphere in school-age children and adolescents with ASD and failed to demonstrate significant differences in either the latency or amplitude between TD and ASD children [25], which was not inconsistent with our results in the left hemisphere.

In this study, we showed that the locations of the ECDs for P50m were more anterior in the right hemisphere than in the left hemisphere in both groups (Table 2). This trend is consistent with previous studies that have also demonstrated the asymmetry of $\mathrm{P} 50 \mathrm{~m}$ locations in 6- to 9-year-old TD children [30,66]. With regard to $\mathrm{P} 50 \mathrm{~m}$, the present study is the first to show the P50m source position in young children with ASD and to demonstrate that both TD and ASD children similarly displayed right-sided $\mathrm{P} 50 \mathrm{~m}$ anteriority. With regard to 
$\mathrm{N} 100 \mathrm{~m}$, a previous study demonstrated right-sided N100m anteriority in 8- to 15-year-old TD children, whereas children with ASD did not show asymmetry [43]. However, we did not examine the N100m component in this study because of a lower GOF in the ECD estimation in 2- to 5-year-old children [31].

The present study had some general limitations. First, we investigated the $\mathrm{P} 50 \mathrm{~m}$ using only one type of auditory stimulus (the human voice /ne/). Therefore, we cannot determine whether our results are specific to human voice stimuli. Second, we analysed the brain's response to auditory stimuli only. Therefore, we cannot generalise this reduction in leftward brain lateralisation in children with ASD to other sensory modalities [19] or multisensory stimuli [67]. Third, a majority of the children with ASD in the present study were high-functioning subjects and therefore may not represent children with ASD who have a comorbid language disability. Fourth, the participants were monitored using a video camera, which facilitated detection of obvious body movement. The examiner who accompanied the children in the shielded room also instructed the participants to maintain the position of their head throughout the experiment. However, the influence of fine head movements might have been a confounding factor in the present study. Fifth, we employed a cross-sectional rather than a longitudinal design. Future research with a longitudinal design is crucially necessary to provide a better understanding of functional maturations in the auditory cortex in children with ASD. In spite of these limitations, the present study is the first to demonstrate atypical brain maturation related to $\mathrm{P} 50 \mathrm{~m}$ generation in young children with ASD.

\section{Conclusions}

Child-customised MEG devices facilitated the acquisition of whole-brain functional measurements during conscious conditions in young children. Children with ASD (most of whom were high-functioning subjects) showed less leftward lateralisation of the intensity of the P50m component in response to voice stimuli compared with TD children. Regardless of their language development, our results suggest that there is atypical brain function in the auditory cortex of young children with ASD.

\section{Additional file}

Additional file 1: Figure S1. Summary of the hierarchical regression analysis for the log-transformed intensity of P50m. The performance of children with ASD and young TD children on each K-ABC subtest (mean score \pm SD) is shown for tests applicable to all ages. An unpaired $t$-test revealed significantly lower performance in children with ASD compared with TD children on the riddle task, which tests "language conceptual inference ability". * $P<0.05$. Table $\mathbf{S 1}$. Summary of the hierarchical regression analysis for the log-transformed intensity of P50m. ${ }^{*} p<.025$.
Table S2. Summary of the hierarchical regression analysis for P50m latency. ${ }^{*} p<.025$.

\section{Abbreviations}

AEF: Auditory evoked magnetic field; ASD: Autism spectrum disorder; TD: Typical developing; ECD: Equivalent current dipole;

EEG: Electroencephalography; ISI: Interstimulus interval; K-ABC: Kaufman Assessment Battery for Children; MEG: Magnetoencephalography;

SLI: Specific language impairment.

\section{Competing interests}

The authors declare no competing interests.

\section{Authors' contributions}

YY participated in the conception and design of the study, performed the statistical analysis and wrote the first draft of the manuscript. MK participated in the conception design, and coordination of the study and wrote the final draft of the manuscript. KS and SU participated in the conception of the study and operated the child-customised MEG system. TM, YN, TTa and MS recruited participants in this study from their facilities. YO, TTs, MO and GBR participated in the conception of the study and advised $Y Y$ regarding the assessment of the ASD and analysis. YH advised KS and SU regarding the operation of the child-customised MEG system. HH and YM participated in the conception of this study and took chief responsibility for the study. All the authors read and approved the final manuscript.

\section{Acknowledgements}

This study was supported by Grant-in-Aid for Specially Promoted (research no. 24000012), the Hokuriku Innovation Cluster for Health Science (MEXT Program for Fostering Regional Innovation) and the Strategic Research Program for Brain Sciences from MEXT, Japan.

\section{Author details}

${ }^{1}$ Research Centre for Child Mental Development, Kanazawa University, Kanazawa, Japan. 'Department of Psychiatry and Neurobiology, Graduate School of Medical Science, Kanazawa University, Kanazawa, Japan. ${ }^{3}$ Higher Brain Functions and Autism Research, Department of Child Development, United Graduate School of Child Development, Osaka University, Kanazawa University and Hamamatsu University School of Medicine, Osaka University, Osaka, Japan. ${ }^{4}$ International Education Centre, Kyushu University, Fukuoka, Japan. ${ }^{5}$ Department of Anaesthesiology, Graduate School of Medical Science, Kanazawa University, Kanazawa, Japan. ${ }^{6}$ Department of MEG, Yokogawa Electric Corporation, Tokyo, Japan. ${ }^{7}$ Department of Neuropsychiatry, University of Toyama, Toyama, Japan.

Received: 17 January 2013 Accepted: 12 September 2013

Published: 8 October 2013

\section{References}

1. Baird G, Simonoff E, Pickles A, Chandler S, Loucas T, Meldrum D, Charman T: Prevalence of disorders of the autism spectrum in a population cohort of children in South Thames: the Special Needs and Autism Project (SNAP). Lancet 2006, 368:210-215.

2. Lord C: Epidemiology: How common is autism? Nature 2011, 474:166-168.

3. Lord C, Spence SJ, Moldin SO, Rubenstein JLR: Autism spectrum disorders: Phenotype and diagnosis. Boca Raton, FL, US: CRC Press; 2006.

4. De Giacomo A, Fombonne E: Parental recognition of developmental abnormalities in autism. Eur Child Adolesc Psychiatry 1998, 7:131-136.

5. Wetherby AM, Woods J, Allen L, Cleary J, Dickinson H, Lord C: Early indicators of autism spectrum disorders in the second year of life. J Autism Dev Disord 2004, 34:473-493.

6. Kamio Y, Robins D, Kelley E, Swainson B, Fein D: Atypical lexical/semantic processing in high-functioning autism spectrum disorders without early language delay. J Autism Dev Disord 2007, 37:1116-1122.

7. Kikuchi M, Shitamichi K, Yoshimura Y, Ueno S, Remijn GB, Hirosawa T, Munesue T, Tsubokawa T, Haruta Y, Oi M, et al: Lateralized theta wave connectivity and language performance in 2- to 5-year-old children. J Neurosci 2011, 31:14984-14988. 
8. Wada JA, Clarke R, Hamm A: Cerebral hemispheric asymmetry in humans. Cortical speech zones in 100 adults and 100 infant brains. Arch Neurol 1975, 32:239-246.

9. Chi JG, Dooling EC, Gilles FH: Left-right asymmetries of the temporal speech areas of the human fetus. Arch Neurol 1977, 34:346-348.

10. Balsamo LM, Xu B, Grandin CB, Petrella JR, Braniecki SH, Elliott TK, Gaillard WD: A functional magnetic resonance imaging study of left hemisphere language dominance in children. Arch Neurol 2002, 59:1168-1174.

11. Dehaene-Lambertz G, Dehaene S, Hertz-Pannier L: Functional neuroimaging of speech perception in infants. Science 2002, 298:2013-2015.

12. Ahmad Z, Balsamo LM, Sachs BC, Xu B, Gaillard WD: Auditory comprehension of language in young children: neural networks identified with fMRI. Neurology 2003, 60:1598-1605.

13. Pena M, Maki A, Kovacic D, Dehaene-Lambertz G, Koizumi H, Bouquet F, Mehler J: Sounds and silence: an optical topography study of language recognition at birth. Proc Natl Acad Sci U S A 2003, 100:11702-11705.

14. Everts R, Lidzba K, Wilke M, Kiefer C, Mordasini M, Schroth G, Perrig W, Steinlin $\mathrm{M}$ : Strengthening of laterality of verbal and visuospatial functions during childhood and adolescence. Hum Brain Mapp 2009, 30:473-483.

15. Herbert MR, Ziegler DA, Deutsch CK, O'Brien LM, Kennedy DN, Filipek PA, Bakardjiev Al, Hodgson J, Takeoka M, Makris N, et al: Brain asymmetries in autism and developmental language disorder: a nested whole-brain analysis. Brain 2005, 128:213-226.

16. Gage NM, Juranek J, Filipek PA, Osann K, Flodman P, Isenberg AL, Spence $M A$ : Rightward hemispheric asymmetries in auditory language cortex in children with autistic disorder: an MRI investigation. J Neurodev Disord 2009, 1:205-214

17. Samson F, Mottron L, Soulieres I, Zeffiro TA: Enhanced visual functioning in autism: An ALE meta-analysis. Hum Brain Mapp 2012, 33:1553-81.

18. Eyler LT, Pierce $K$, Courchesne E: A failure of left temporal cortex to specialize for language is an early emerging and fundamental property of autism. Brain 2012, 135:949-960.

19. Fiebelkorn IC, Foxe JJ, McCourt ME, Dumas KN, Molholm S: Atypical category processing and hemispheric asymmetries in high-functioning children with autism: Revealed through high-density EEG mapping. Cortex 2013, 49:1259-67.

20. Redcay $E$, Courchesne E: Deviant functional magnetic resonance imaging patterns of brain activity to speech in 2-3-year-old children with autism spectrum disorder. Biol Psychiatry 2008, 64:589-598.

21. Kikuchi M, Shitamichi K, Yoshimura Y, Ueno S, Hiraishi H, Hirosawa T, Munesue T, Nakatani H, Tsubokawa T, Haruta Y, et al: Altered brain connectivity in 3-to 7-year-old children with autism spectrum disorder. Neurolmage: Clinical 2013, 2:394-401.

22. Rojas DC, Camou SL, Reite ML, Rogers SJ: Planum temporale volume in children and adolescents with autism. J Autism Dev Disord 2005, 35:479-486.

23. Potter D, Summerfelt A, Gold J, Buchanan RW: Review of clinical correlates of P50 sensory gating abnormalities in patients with schizophrenia. schizophr Bull 2006, 32:692-700

24. Ponton C, Eggermont JJ, Khosla D, Kwong B, Don M: Maturation of human central auditory system activity: separating auditory evoked potentials by dipole source modeling. Clin Neurophysio/ 2002, 113:407-420.

25. Oram Cardy JE, Ferrari P, Flagg EJ, Roberts W, Roberts TP: Prominence of M50 auditory evoked response over M100 in childhood and autism. Neuroreport 2004, 15:1867-1870.

26. Gilley PM, Sharma A, Dorman M, Martin K: Developmental changes in refractoriness of the cortical auditory evoked potential. Clin Neurophysio 2005, 116:648-657.

27. Wunderlich JL, Cone-Wesson BK, Shepherd R: Maturation of the cortical auditory evoked potential in infants and young children. Hear Res 2006, 212:185-202.

28. Edgar JC, Huang MX, Weisend MP, Sherwood A, Miller GA, Adler LE, Canive JM: Interpreting abnormality: an EEG and MEG study of P50 and the auditory paired-stimulus paradigm. Biol Psychol 2003, 65:1-20.

29. Pihko E, Kujala T, Mickos A, Alku P, Byring R, Korkman M: Language impairment is reflected in auditory evoked fields. Int J Psychophysio/ 2008, 68:161-169.

30. Pihko E, Mickos A, Kujala T, Pihlgren A, Westman M, Alku P, Byring R, Korkman M: Group intervention changes brain activity in bilingual language-impaired children. Cereb Cortex 2007, 17:849-858.

31. Yoshimura Y, Kikuchi M, Shitamichi K, Ueno S, Remijn GB, Haruta Y, Oi M, Munesue T, Tsubokawa T, Higashida $H$, et al: Language performance and auditory evoked fields in 2- to 5-year-old children. Eur J Neurosci 2012, 35:644-650.

32. Kaufman A, Kaufman N: Kaufman Assessment Battery for Children: Administration and Scoring Manual. Circle Pines, MN: American Guidance Service; 1983.

33. Hertrich I, Mathiak K, Lutzenberger W, Ackermann H: Differential impact of periodic and aperiodic speech-like acoustic signals on magnetic M50/ M100 fields. Neuroreport 2000, 11:4017-4020.

34. Chait M, Simon JZ, Poeppel D: Auditory M50 and M100 responses to broadband noise: functional implications. Neuroreport 2004, 15:2455-2458.

35. Salamy A: Commissural transmission: maturational changes in humans. Science 1978, 200:1409-1411.

36. Huttenlocher PR, Dabholkar AS: Regional differences in synaptogenesis in human cerebral cortex. J Comp Neurol 1997, 387:167-178.

37. Moore JK, Guan YL: Cytoarchitectural and axonal maturation in human auditory cortex. J Assoc Res Otolaryngol 2001, 2:297-311.

38. Brauer J, Anwander A, Friederici AD: Neuroanatomical prerequisites for language functions in the maturing brain. Cereb Cortex 2011, 21:459-466.

39. Pujol J, Soriano-Mas C, Ortiz H, Sebastian-Galles N, Losilla JM, Deus J: Myelination of language-related areas in the developing brain. Neurology 2006, 66:339-343.

40. Seri S, Cerquiglini A, Pisani F, Curatolo P: Autism in tuberous sclerosis: evoked potential evidence for a deficit in auditory sensory processing. Clin Neurophysiol 1999, 110:1825-1830.

41. Gage NM, Siegel B, Callen M, Roberts TP: Cortical sound processing in children with autism disorder: an MEG investigation. Neuroreport 2003, 14:2047-2051

42. Oram Cardy JE, Flagg EJ, Roberts W, Brian J, Roberts TP: Magnetoencephalography identifies rapid temporal processing deficit in autism and language impairment. Neuroreport 2005, 16:329-332.

43. Schmidt GL, Rey MM, Oram Cardy JE, Roberts TP: Absence of M100 source asymmetry in autism associated with language functioning. Neuroreport 2009, 20:1037-1041.

44. Roberts TP, Khan SY, Rey M, Monroe JF, Cannon K, Blaskey L, Woldoff S, Qasmieh S, Gandal M, Schmidt GL, et al: MEG detection of delayed auditory evoked responses in autism spectrum disorders: towards an imaging biomarker for autism. Autism Res 2010, 3:8-18.

45. Roberts TP, Cannon KM, Tavabi K, Blaskey L, Khan SY, Monroe JF, Qasmieh S, Levy SE, Edgar JC: Auditory Magnetic Mismatch Field Latency: A Biomarker for Language Impairment in Autism. Biol Psychiatry 2011.

46. Lord C, Rutter M: DiLavore P, Risi S: Autism Diagnostic Observation Schedule. Los Angeles, CA: Western Psychological Services; 1999.

47. Wing L, Leekam SR, Libby SJ, Gould J, Larcombe M: The Diagnostic Interview for Social and Communication Disorders: background, interrater reliability and clinical use. J Child Psychol Psychiatry 2002, 43:307-325.

48. Kikuchi M, Yoshimura Y, Shitamichi K, Ueno S, Hirosawa T, Munesue T, Ono Y, Tsubokawa T, Haruta Y, Oi M, et al: A custom magnetoencephalography device reveals brain connectivity and high reading/decoding ability in children with autism. Sci Rep 2013, 3:1139.

49. Johnson BW, Crain S, Thornton R, Tesan G, Reid M: Measurement of brain function in pre-school children using a custom sized whole-head MEG sensor array. Clin Neurophysiol 2010, 121:340-349.

50. Cook HM: The sentence-final particle ne as a tool for cooperation in Japanese convcrsation. Stanford: The Stanford Linguistic Association; 1990.

51. Anderson $\mathrm{V}$, Hiramoto $\mathrm{M}$, Wong A: Prosodic analysis of the interactional particle ne in Japanese gendered speech. Japanese/Korean Linguistics 2007, 15:43-54.

52. Kajikawa S, Amano S, Kondo T: Speech overlap in Japanese mother-child conversations. J Child Lang 2004, 31:215-230.

53. Squires T: A discourse anlysis of the Japanese particle sa. Pragmatics 2009, $4: 1-29$.

54. Elberling C, Bak C, Kofoed B, Lebech J, Saermark K: Auditory magnetic fields from the human cerebral cortex: location and strength of an equivalent current dipole. Acta Neurol Scand 1982, 65:553-569.

55. Tsuneishi $S$, Casaer $P$ : Stepwise decrease in VEP latencies and the process of myelination in the human visual pathway. Brain Dev 1997, 19:547-551.

56. Roncagliolo M, Benitez J, Equibar JR: Progressive deterioration of central components of auditory brainstem responses during postnatal development of the myelin mutant taiep rat. Audiol Neurootol 2000, 5:267-275.

57. Lee DL, Strathmann FG, Gelein R, Walton J, Mayer-Proschel M: Iron deficiency disrupts axon maturation of the developing auditory nerve. J Neurosci 2012, 32:5010-5015 
58. Lang EJ, Rosenbluth J: Role of myelination in the development of a uniform olivocerebellar conduction time. J Neurophysiol 2003, 89:2259-2270.

59. Krubitzer L, Kahn DM: Nature versus nurture revisited: an old idea with a new twist. Prog Neurobiol 2003, 70:33-52.

60. Ben Bashat D, Kronfeld-Duenias V, Zachor DA, Ekstein PM, Hendler T, Tarrasch R, Even A, Levy Y, Ben Sira L: Accelerated maturation of white matter in young children with autism: a high b value DWI study. Neuroimage 2007, 37:40-47.

61. Flagg EJ, Cardy JE, Roberts W, Roberts TP: Language lateralization development in children with autism: insights from the late field magnetoencephalogram. Neurosci Lett 2005, 386:82-87.

62. De Fosse L, Hodge SM, Makris N, Kennedy DN, Caviness VS Jr, McGrath L, Steele S, Ziegler DA, Herbert MR, Frazier JA, et al: Language-association cortex asymmetry in autism and specific language impairment. Ann Neurol 2004, 56:757-766.

63. Reite $\mathbf{M}$, Teale P, Zimmerman J, Davis K, Whalen J: Source location of a 50 msec latency auditory evoked field component. Electroencephalogr Clin Neurophysiol 1988, 70:490-498.

64. Sharma A, Kraus N, McGee TJ, Nicol TG: Developmental changes in P1 and $\mathrm{N} 1$ central auditory responses elicited by consonant-vowel syllables. Electroencephalogr Clin Neurophysiol 1997, 104:540-545.

65. Oram Cardy JE, Flagg EJ, Roberts W, Roberts TP: Auditory evoked fields predict language ability and impairment in children. Int $J$ Psychophysiol 2008, 68:170-175.

66. Pihko E, Kujala T, Mickos A, Antell H, Alku P, Byring R, Korkman M: Magnetic fields evoked by speech sounds in preschool children. Clin Neurophysiol 2005, 116:112-119.

67. Brandwein AB, Foxe JJ, Butler JS, Russo NN, Altschuler TS, Gomes H, Molholm S: The Development of Multisensory Integration in HighFunctioning Autism: High-Density Electrical Mapping and Psychophysical Measures Reveal Impairments in the Processing of Audiovisual Inputs. Cereb Cortex 2012.

doi:10.1186/2040-2392-4-38

Cite this article as: Yoshimura et al:: Atypical brain lateralisation in the auditory cortex and language performance in 3- to 7-year-old children with high-functioning autism spectrum disorder: a child-customised magnetoencephalography (MEG) study. Molecular Autism 2013 4:38.

\section{Submit your next manuscript to BioMed Central and take full advantage of:}

- Convenient online submission

- Thorough peer review

- No space constraints or color figure charges

- Immediate publication on acceptance

- Inclusion in PubMed, CAS, Scopus and Google Scholar

- Research which is freely available for redistribution 